\title{
An Algorithm for Recovering Camouflage Errors on Moving People
}

\author{
D. Conte, P. Foggia, G. Percannella, F. Tufano, and M. Vento \\ Dipartimento di Ingegneria dell'Informazione ed Ingegneria Elettrica \\ Via Ponte Don Melillo, 84084 Fisciano (SA), Italy \\ \{dconte,pfoggia, pergen, ftufano, mvento\}@unisa.it
}

\begin{abstract}
In this paper we present a model-based algorithm working as a post-processing phase of any foreground object detector. The model is suited to recover camouflage errors producing the segmentation of an entity in small and unconnected parts. The model does not require training procedures, but only information about the estimated size of the person, obtainable when an inverse perspective mapping procedure is used.

A quantitative evaluation of the effectiveness of the method, used after four well known moving object detection algorithms has been carried out. Performance are given on a variety of publicly available databases, selected among those presenting highly camouflaged objects in real scenes referring to both indoor and outdoor environments.
\end{abstract}

\section{Introduction}

Most of video analysis applications require the extraction of the moving objects from the scene, so as to apply further processing aimed to classify them into different categories (persons, vehicles, animals, bags or luggage), or to characterize the trajectories traced in the environment. Both these elaborations are necessary processing steps toward the understanding of the events occurring in the scene. Of course, the semantic analysis is unavoidably affected by any error occurring in the detection phase, as the fragmentation of a single object into parts (for instance, a person into pieces of body), or the merging of close objects into a bigger one.

In this complex application framework, starting from the first approaches to the problem, background subtraction is being considered as a simple and powerful technique for detecting the moving objects as opposed to the static elements that are part of the observed scene. Although many related issues have been receiving further research attention, and some problems are still considered open, the background subtraction techniques are gaining popularity among the object detection algorithms.

Experimentation over the years, highlighted that the background subtraction technique is subject to a set of well known problems, categorized in [9]; consequently, most of the algorithms proposed up to now, have been devised so as to face the above cited problems. A noteworthy exception is constituted by 
camouflage: an intrinsic and hardly faceable problem occurring when the pixel characteristics of a foreground object are too similar to the background to be discerned, as happens when a person is wearing clothes having similar colors to the background. The effect is that the difference of these pixels from the background model is under the threshold, and consequently incorrectly considered as foreground pixels.

Camouflage has comparatively received less attention than the other problems, probably because most of detection methods operate at a pixel level, where there is not enough information to effectively tackle this problem. So most authors either ignore the issue, testing their detection algorithms in contexts where camouflage is unlikely, or assume that later processing phases will be able to correct the anomalies induced by camouflage.

Among the papers specifically devoted to the camouflage problem, Tankus and Yeshurum [8] propose the use of an operator to enhance areas whose shading corresponds to a convex object to separate such areas from a "flat" background with similar intensity and texture. However the method is not suitable for environments in which the background also contains convex objects, and does not work well for objects with dark colors.

The paper by Harville et al. 4 is representative of an approach to the problem that involves the use of depth information to detect camouflaged objects. The authors also evaluate other popular video analysis methods proposed in the literature, maintaining that among the considered systems, only the ones incorporating depth information are able to deal with camouflage. While the use of depth information can surely improve the detection performance of a video analysis system, it has a non negligible computational cost and, more important, it precludes the use of the legacy cameras often already installed for applications such as video-surveillance or traffic monitoring.

The paper by Boult et al. [1] is devoted to intentional camouflage, and uses background subtraction with two thresholds: a larger and a smaller one, used to respectively detect pixels that are certainly in the foreground, or either part of the background or a camouflaged part of the foreground. The regions detected using the two thresholds are then grouped using suitable conditions to form the so called "Quasi Connected Components" to recover the split of camouflaged objects.

The approach proposed by TrakulPong and Bowden [10] is instead based on a simple model of the shape, integrated in the tracking phase; it builds a statistical model of the shape of the tracked object, and when an abrupt shape change occurs, the algorithm assumes it is due to camouflage and tries to match the object image at the previous frame to restore the correct shape.

The paper by Guo et al. 3. proposes to address the camouflage problem by performing a temporal averaging of the frames before computing or updating the background model. The idea is that this way, the model will have a smaller variance and so a smaller detection threshold can be used. However, as the experiments performed by the authors show, the method has problems with slowly moving objects. 
In this paper we propose a method for correcting the errors typically generated in the detection phase of a background subtraction procedure, in presence of camouflage; its generality allows the user to apply it as a post processing module operating after a generic object detection algorithm.

The errors, consisting in the fragmentation of the actual object in the scene, are detected and corrected by a grouping phase performed on the basis of a model of the shape to be recognized, that in our system are isolated people. Once the object have been detected, a set of merges of adjacent objects are performed in the case that, after fusion the obtained object is more likely to be a person. For the sake of notational simplicity, hereinafter we denote with blobs all those objects generated as output by the detection phase, independently of the fact that they are actually persons, fragments of them or unanimated objects. The algorithm has been devised so as to make it possible the recursive merging of blobs, so as to allow the possibility of recovering highly critical situations caused by camouflage, as the split of a single person in a plurality of small parts, otherwise considered as noise.

The algorithm has been widely experimented applying it on the output of four well known detection algorithms, over a wide video database publicly available, including indoor and outdoor scenes.

\section{Proposed Method}

As anticipated in the introduction, the camouflage problem causes that the foreground mask of a person is split into two or more foreground blobs so generating plenty of different configurations. The Fig. 1 shows the output of some different foreground detection algorithms on various video sequences. It is evident from the picture that, even recognizable by a human being, the obtained configurations of blobs are far to be considered as ideal. A great effort must be done
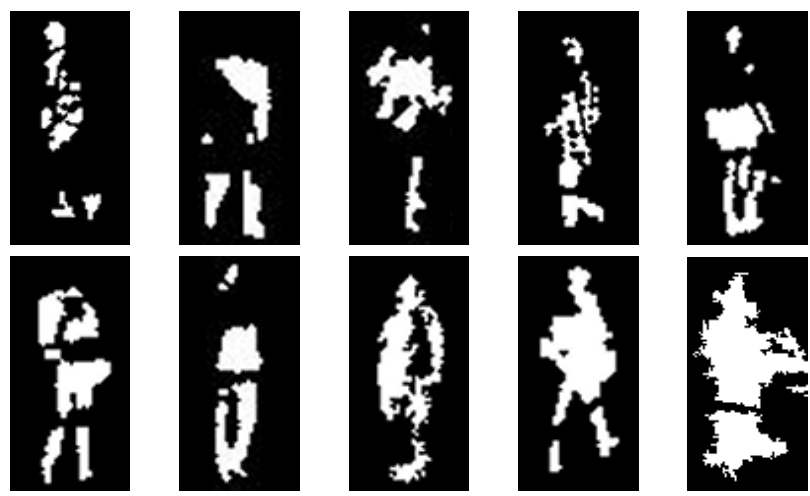

Fig. 1. Examples of errors generated in the detection phase of a background subtraction procedure in presence of camouflage. In all cases a person is detected as a set of separated blobs. 
to process them so as to obtain, for each person a single blob. The problem of recovering such kind of errors is equivalent to the problem of finding how several foreground blobs can be grouped together in order to suitably represent the object of interest, in our case a person. By examining a single blob it is not possible to determine if it can be considered as a part of a larger object or if it is spuriously generated during the foreground detection process. So, to reconstruct the objects affected by camouflage errors we need to define a model of the desired object and a procedure for suitably grouping the obtained blobs, so as to adequately fit the model.

In this paper we focus our attention to the detection of moving people, but the underlying idea can be generalized to the detection of other kinds of objects (as cars, animals, etc.).

Obviously the model must be carefully defined: a too detailed model would result in many missed detections, while on the contrary a too general one would cause the spurious generation of a plenty of false positive errors (blobs due to detection errors grouped to form, erroneously, objects of interest).

The chosen model starts from the simple consideration, that, as shown in Fig. 1, however the parts are arranged, they fall into an ideal box representing a person. It is worth noting that the model cannot be defined on the basis of the size expressed in pixels. The perspective causes, in fact, that a same configuration of pixels represents an object of different actual size depending on the distance from the camera. Therefore, the model be must defined in terms of actual size, and a suitably defined Inverse Perspective Mapping procedure must be used to pass from measures in the pixel space to actual ones.

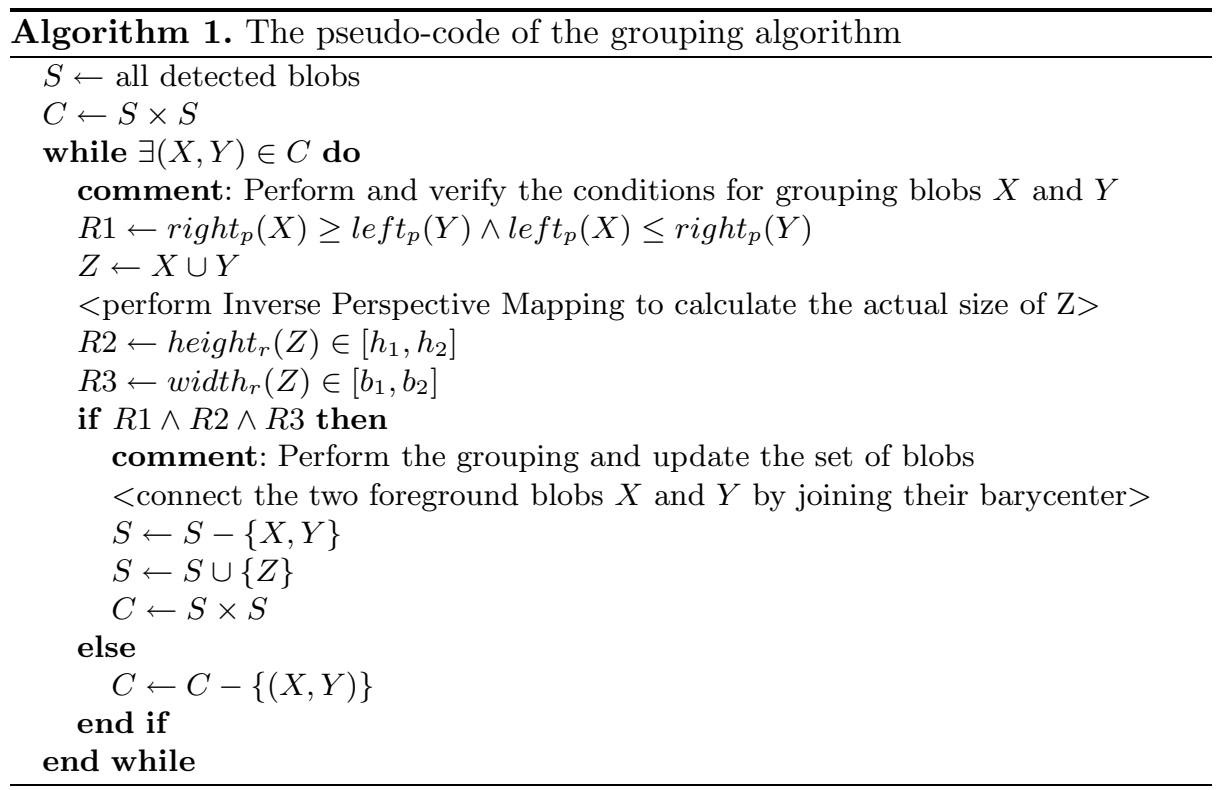






Fig. 2. The possible configurations of the overlaps between the projections of two boxes on the horizontal axis

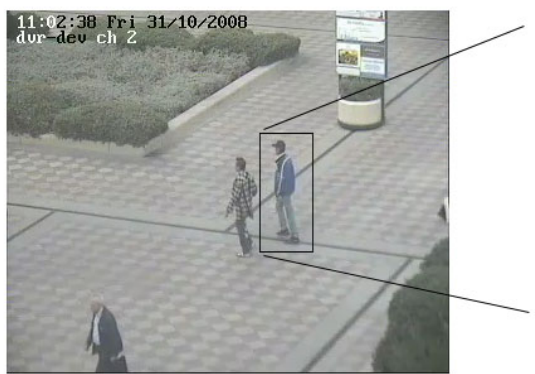

a)

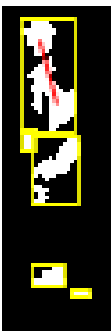

d)

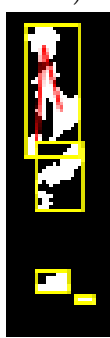

e)
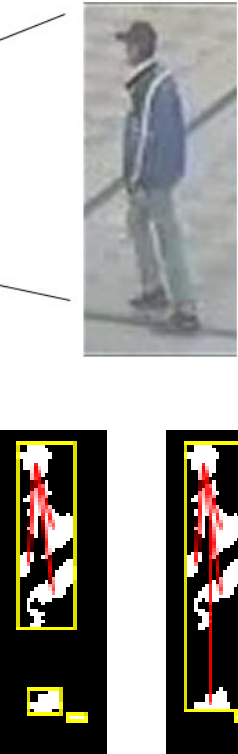

f)

g)

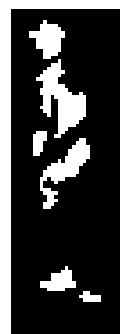

b)

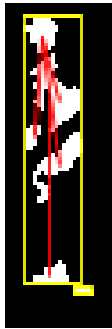

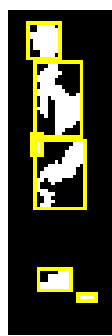

c)

Fig. 3. An example of the algorithm's processing: a) original frame and the portion under analysis; b) the resulting foreground detection and the c) resulting bounding boxes; d), e), f), g), h) the steps of the algorithms on the considered portion of the frame

The adopted model represents a person as a box defined by four parameters $h_{1}, h_{2}, b_{1}$ and $b_{2}$ that, respectively, are the minimum and maximum actual height and the minimum and maximum actual width.

The pseudo-code of the algorithm is sketched in Algorithm 1. The aim of the algorithm is to group two or more blobs in order to form a unique blob representing a person (according to the defined model). Coherently with the adopted representation of a person, the algorithm represents each blob by its 
bounding box. The procedure operates by repeatedly merging couples of blobs into larger ones until the new blob best fits the defined model of person. Two blobs are grouped if and only if all the following conditions are verified:

R1. The projection on the horizontal axis of the bounding box of the considered blobs are overlapped (see Fig. 2). Note that the coordinates of the two boxes $\left(\operatorname{left}_{p}(X), \operatorname{right}_{p}(X), \operatorname{left}_{p}(Y), \operatorname{right}_{p}(Y)\right.$ in Algorithm 1) are expressed in pixels.

R2. The actual height, in meters, of the box grouping the two blobs $\left(\right.$ height $_{r}(Z)$ in Algorithm 1) is included between $h_{1}$ and $h_{2}$.

R3. The actual width, in meters, of the box grouping the two blobs $\left(\right.$ width $_{r}(Z)$ in Algorithm 1) is included between $b_{1}$ and $b_{2}$.

To verify the last two conditions, we firstly build the box grouping the considered blobs starting from the corresponding boxes by their pixels coordinates. Then, the Inverse Perspective Mapping is applied to the constructed box in order to determine its real size.

It is important to highlight that the proposed method is not computationally expensive because the number of detected boxes per frame is never greater than one or two dozens.

In Fig. 3 an example of the application of the algorithm is sketched.

\section{Experimental Results}

The experimental validation of the proposed method has been carried out by evaluating the performance improvements obtained when it is used as a postprocessing on the output of four well known techniques of foreground detection; in particular:

- the Mixture of Gaussians (from now on called $M O G$ ), in the version proposed by Kaewtrakulpong and Bowden in [5];

- the Enhanced Background Subtraction (from now on EBS), proposed by Conte et al. in [2];

- the Self-Organizing Background Subtraction (from now on $S O B S$ ), proposed by Maddalena and Petrosino in [7;

- the Statistical Background Algorithm (from now on $S B A$ ), proposed by Li et al. in [6].

The performance is measured by using the $f$-score index, defined as the harmonic mean of precision and recall, according to the following formulas:

$$
\text { precision }=\frac{T P}{T P+F P} \quad \text { recall }=\frac{T P}{T P+F N}
$$

In the previous formulas, the true positive (TP), false positive $(\mathrm{FP})$ and false negative $(\mathrm{FN})$ are given by: 


$$
\begin{gathered}
T P=\sum_{g \in G} \sum_{d \in D} \frac{|g \cap d|}{|g \cup d|} \quad F P=\sum_{d \in D} \frac{|d|-\max _{g \in G}|d \cap g|}{|d|} \\
F N=\sum_{g \in G} \frac{|g|-\max _{d \in D}|d \cap g|}{|g|}
\end{gathered}
$$

where $G$ is the set of objects of the ground truth and D is the set of objects really detected by the algorithm (each object is represented by its bounding box).

Tests have been done on a dataset of five real video sequences either indoor or outdoor. In Table 1 the main features of the considered videos are reported: the visual properties (number of frames of the sequence, frame rate expressed in fps, resolution), a short description of the content. The dataset has been also characterized in terms of the total number of foreground objects in each sequence and the number of isolated persons. This data allows, on one side, to evaluate the effectiveness of the proposed method in terms of people detected by grouping single pieces and, on the other side, to quantify the overall detection performance (i.e. when in the scene there are also objects the method was not designed to handle, as animals, bags, ...)

The NA1-NA3 videos were acquired by the authors on a large square in different lighting and weather conditions, with several persons walking. The PETS video belongs to the dataset published at the 2006 edition of the PETS workshop and contains a scene framed within a railway station. The MSA sequence, presented in [7], refers to an indoor scene.

Table 2 reports the performance of the considered four algorithms when the proposed method is adopted or not together with the relative improvements. The results are given in two cases: respectively, when all the objects in the dataset or only people objects are considered.

Table 1. Main features of the employed dataset: the properties (number of frames of the sequence, frame rate expressed in fps, resolution), a short description of the content and of the total number of objects and the number of isolated persons

\begin{tabular}{|c||c|l|c|c|}
\hline $\begin{array}{c}\text { Video } \\
\text { ID }\end{array}$ & Properties & Description & $\begin{array}{c}\text { \# of } \\
\text { objects }\end{array}$ & $\begin{array}{c}\text { \# of people } \\
\text { objects }\end{array}$ \\
\hline \hline NA1 & $9^{\prime} 365,25,352 \times 288$ & $\begin{array}{l}\text { outdoor, sunny, very dark shad- } \\
\text { ows }\end{array}$ & $19^{\prime} 093$ & $17^{\prime} 875(93.6 \%)$ \\
\hline NA2 & $4^{\prime} 575,25,352 \times 288$ & $\begin{array}{l}\text { outdoor, cloudy, very high cam- } \\
\text { ouflage, few shadows }\end{array}$ & $9^{\prime} 333$ & $7^{\prime} 651(82.0 \%)$ \\
\hline NA3 & $21^{\prime} 000,25,352 \times 288$ & $\begin{array}{l}\text { outdoor, late afternoon, high } \\
\text { camouflage, very long shadows }\end{array}$ & $20^{\prime} 568$ & $18^{\prime} 303(89.0 \%)$ \\
\hline PETS & $2^{\prime} 556,25,768 \times 576$ & indoor, reflections & $5^{\prime} 779$ & $4^{\prime} 823(83.5 \%)$ \\
\hline MSA & $528,30,352 \times 288$ & indoor, vertical shadows & 685 & $329(48 \%)$ \\
\hline
\end{tabular}


Table 2. Object detection performance given in terms of $f$-score obtained considering when all the objects of the dataset (rows denoted with all), or only people objects (rows denoted with people). The columns before and after show the performance of the original algorithms without and with the proposed post-processing, respectively.

\begin{tabular}{|c|c|c|c|c|c|c|c|c|c|c|c|c|c|}
\hline \multirow{2}{*}{\begin{tabular}{|c|} 
Video \\
ID \\
\end{tabular}} & \multirow{2}{*}{$\begin{array}{c}\text { Object } \\
\text { type }\end{array}$} & \multicolumn{3}{|c|}{ EBS } & \multicolumn{3}{|c|}{ MOG } & \multicolumn{3}{|c|}{ SBA } & \multicolumn{3}{|c|}{ SOBS } \\
\hline & & bef. & aft. & imp. & bef. & aft. & imp. & bef. & aft. & imp. & bef. & aft. & imp. \\
\hline \multirow{2}{*}{ NA1 } & all & \begin{tabular}{|l|}
.671 \\
\end{tabular} & .673 & $0.3 \%$ & .657 & .668 & $2.0 \%$ & .220 & .304 & $38.5 \%$ & .530 & .573 & $8.0 \%$ \\
\hline & people & .732 & .747 & $2.1 \%$ & .646 & .645 & $0.1 \%$ & .212 & .312 & $46,9 \%$ & .516 & .591 & $14.6 \%$ \\
\hline \multirow{2}{*}{ NA2 } & all & .775 & .875 & $12.9 \%$ & .671 & .676 & $0.7 \%$ & 256 & .278 & $8.5 \%$ & .434 & .510 & $17.4 \%$ \\
\hline & people & .729 & .873 & $19.8 \%$ & .648 & .663 & $2.3 \%$ & .198 & .269 & $35.9 \%$ & .416 & .449 & $7.9 \%$ \\
\hline \multirow{2}{*}{ NA3 } & $\overline{\text { all }}$ & .555 & .693 & $24.7 \%$ & .644 & .649 & $0.7 \%$ & .204 & .227 & $10.9 \%$ & .287 & .420 & $46.4 \%$ \\
\hline & people & .554 & .705 & $27.1 \%$ & .646 & .656 & $1.5 \%$ & .202 & .229 & $13.3 \%$ & .304 & .430 & $41.1 \%$ \\
\hline \multirow{2}{*}{ PETS } & all & .773 & .801 & $3.6 \%$ & .753 & .651 & $-13.4 \%$ & .645 & .635 & $-1.5 \%$ & .582 & .606 & $4.0 \%$ \\
\hline & people & .724 & .818 & $13.0 \%$ & .623 & .643 & $3.2 \%$ & .423 & .424 & $0.1 \%$ & .544 & .597 & $9.8 \%$ \\
\hline \multirow{2}{*}{ MSA } & $\overline{\text { all }}$ & .847 & .904 & $6.6 \%$ & .520 & .539 & $3.5 \%$ & .163 & .206 & $26.4 \%$ & .850 & .850 & - \\
\hline & people & .839 & .921 & $9.7 \%$ & .542 & .565 & $4.3 \%$ & 613 & .622 & $1.5 \%$ & .816 & .828 & $1.5 \%$ \\
\hline
\end{tabular}

If we consider the results reported in Table 2 it is possible to note that in the large majority of cases the use of the proposed method improves the object detection performance.

By looking at the data in Table 2 with respect to the video sequence it is possible to consider that according to the obtained performance improvements, the video sequences can be roughly divided in three groups. The first group, composed by the NA2 and NA3 videos, is the one on which almost all the algorithms reach the highest improvements, ranging from about $10 \%$ to over $45 \%$, with the exception of MOG whose behavior will be deeper discussed in the following. The second group, containing the NA1 and the MSA sequences, presents moderate improvements, while, finally, the performance is generally low on PETS. It is worth pointing out that the behavior of the algorithms on the above defined groups can be related to the characteristics of the videos as described in the Table 1. The limitation of color gamut in the video sequences belonging to the first group, due to the poor scene illumination (cloudy in NA2 and late noon in NA3), favors the occurrence of the camouflage errors: in this case all the algorithms significantly benefits from the use of the proposed grouping procedure. Both the video sequences in the second group contain well illuminated scenes, so as that the camouflage problem occurs less frequently, making the improvements provided by our method less evident. A final consideration is about the efficiency of most of the considered object detectors which tends to worsen when the proposed method is applied on the PETS video. This behavior can be explained by considering that these video sequences were framed in a complex indoor environment with artificial lighting and reflective surfaces. These conditions cause that the original detection algorithms produce numerous false blobs, in some cases erroneously grouped by the grouping procedure.

Moreover, if we analyze the data in Table 2 with respect to the original foreground detection algorithm, it is evident that the adoption of the proposed 
Table 3. Absolute number of objects in the dataset associated to persons, split by the considered detection algorithms; performance are given before and after the grouping algorithm

\begin{tabular}{|c|c|c|c|c|c|c|c|c|c|c|c|c|}
\hline \multirow{2}{*}{\begin{tabular}{c|} 
Video \\
ID
\end{tabular}} & \multicolumn{3}{|c|}{ EBS } & \multicolumn{3}{|c|}{ MOG } & \multicolumn{3}{|c|}{$\overline{\text { SBA }}$} & \multicolumn{3}{|c|}{ SOBS } \\
\hline & bef. & after & impr. & bef. & after & impr. & bef. & after & impr. & bef. & after & impr. \\
\hline NA1 & 530 & \begin{tabular}{|l|}
394 \\
\end{tabular} & $26.7 \%$ & 338 & 333 & $1.5 \%$ & 122 & 67 & $45.1 \%$ & 362 & 273 & $24.6 \%$ \\
\hline NA2 & \begin{tabular}{|l|}
419 \\
\end{tabular} & 206 & $50.8 \%$ & 151 & 125 & $17.2 \%$ & 106 & 72 & $32.1 \%$ & 216 & 171 & $20.8 \%$ \\
\hline NA3 & 1043 & 206 & $80.2 \%$ & \begin{tabular}{|l|}
137 \\
\end{tabular} & 129 & $5.8 \%$ & 147 & 81 & $44.9 \%$ & 573 & 395 & $31.1 \%$ \\
\hline PETS & 215 & 147 & $31.6 \%$ & 106 & 79 & $25.5 \%$ & 91 & 76 & $16.5 \%$ & 185 & 149 & $19.5 \%$ \\
\hline MSA & 17 & 5 & $70.6 \%$ & 7 & 6 & $14.3 \%$ & 21 & 19 & $9.5 \%$ & 11 & 9 & $18.2 \%$ \\
\hline
\end{tabular}

grouping procedure after the EBS, the SBA and the SOBS object detection algorithms produces significant improvements with respect to the f-score that in many cases are above $10 \%$. The only exception is represented by the MOG algorithm that does not benefit from it. This behavior can be justified by considering that the MOG technique (with the exception of the PETS video) tends to be less sensitive to camouflage problems than other typical background subtraction algorithms.

It is worth pointing out that the above considerations are generally valid either when all the objects in the dataset are considered or the tests are carried out with respect only to the people objects.

Table 3 reports an evaluation of the reduction of the problem of objects splitting when the proposed method is used or not. The tests were done by considering only the objects that are associated to persons. The results in Table 3 still confirm that the grouping procedure is effective in recovering the split errors due to camouflage: in all the experiments, the proposed method significantly reduces the number of person detected as separated in several fragments.

\section{Conclusions}

In this paper we present a model-based method for removing errors caused by camouflage in the detection of foreground isolated persons for video surveillance applications. The approach is designed to be used as a post-processing phase of a generic background subtraction algorithm.

A wide experimentation confirmed the effectiveness of the method able to significantly improve the performance in the detection of persons. The tests also highlighted that this improvement can sometimes be moderate, expecially when it is used on videos characterized by very complex environments that cause the detection of many false foreground blobs by the original background subtraction algorithm: in some cases, the false blobs may be merged so determining an erroneous detection of persons. This problem has a very poor impact, even if it can possibly be reduced by suitably refining the model. As future work, we 
are going to extend the approach to other application domains, as the traffic monitoring through the definition of suitable models for the objects of interest (i.e. cars, trucks, bus, ...).

\section{References}

1. Boult, T.E., Michaels, R.J., Gao, X., Eckmann, M.: Into the woods: visual surveillance of noncooperative and camouflaged targets in complex outdoor settings. Proceedings of IEEE 89(10), 1382-1402 (2001)

2. Conte, D., Foggia, P., Petretta, M., Tufano, F., Vento, M.: Meeting the Application Requirements of Intelligent Video Surveillance Systems in Moving Object Detection. In: Singh, S., Singh, M., Apte, C., Perner, P. (eds.) ICAPR 2005. LNCS, vol. 3687, pp. 653-662. Springer, Heidelberg (2005)

3. Guo, H., Dou, Y., Tian, T., Zhou, J., Yu, S.: A robust foreground segmentation method by temporal averaging multiple video frames. In: International Conference on Audio, Lenguage and Image Preocessing, pp. 878-882 (2008)

4. Harville, M., Gordon, G., Woodfill, J.: Foreground segmentation using adaptive mixture models in color and depth. In: IEEE Workshop on Detection and Recognition of Events in Video, pp. 3-11 (2001)

5. Kaewtrakulpong, P., Bowden, R.: An improved adaptive background mixture model for realtime tracking with shadow detection. In: Proc. 2nd European Workshop on Advanced Video Based Surveillance Systems, AVBS 2001, Video Based Surveillance Systems: Computer Vision and Distributed Processing (2001)

6. Li, L., Huang, W., Gu, I.Y.H., Tian, Q.: Foreground object detection from videos containing complex background. In: Proceedings of the eleventh ACM international conference on Multimedia (2003)

7. Maddalena, L., Petrosino, A.: A self-organizing approach to background subtraction for visual surveillance applications. IEEE Transactions on Image Processing 17(7), 1168-1177 (2008)

8. Tankus, A., Yeshurum, Y.: Convexity-based visual camouflage breaking. Computer Vision and Image Understanding 82, 208-237 (2001)

9. Toyama, K., Krumm, J., Brumitt, B., Meyers, B.: Wallflower: Principles and practice of background maintenance. In: Proc. IEEE Int. Conf. Computer Vision, vol. 1, pp. 255-261 (1999)

10. TrakulPong, P.K., Bowden, R.: A real time adaptative visual surveillance system for tracking low-resolution colour targhets in dynamically changing scenes. Image and Vision Computing 17, 913-929 (2003) 